\title{
Sincronía y diacronía del posgrado en educación especial de la Facultad de Estudios Superiores Zaragoza (FESZ): balance y perspectivas
}

\author{
Guadalupe Acle Tomasini ${ }^{\text {*1; }}$ Rosalinda Lozada García ${ }^{2}$; Laura María Martínez Basurto ${ }^{\text {; }}$ \\ Thamir D.D. Durán Fonseca ${ }^{4}$ \\ ${ }^{1}$ http://orcid.org/0000-0002-7292-3870, ${ }^{2}$ http://orcid.org/0000-0003-3287-6140, ${ }^{3}$ http://orcid.org/0000-0002-3045-9317, \\ ${ }^{4} \mathrm{http}$ //orcid.org/0000-0002-3604-2662-3870, 1,2,3,4 Facultad de Estudios Superiores Zaragoza, Universidad Nacional Autónoma \\ de México, México
}

Citar como: Acle G., Lozada, R., Martínez, L., Durán, T. (2021). Sincronía y Diacronía del Posgrado en Educación Especial de la Facultad de Estudios Superiores Zaragoza (FESZ): Balance y Perspectivas. Revista Digital de Investigación en Docencia Universitaria, 16(1), e1573. https://doi.org/10.19083/ridu.2022.1573

Recibido: 03/12/21. Revisado: 07/12/21. Aceptado: 15/01/22. Publicado: 25/02/22.

\section{Resumen}

Introducción: Diseñar un plan de estudios de posgrado es una tarea relevante en las facultades de estudios superiores debido a lo que en el futuro se espera en la formación de sus estudiantes. Instrumentarlo e irlo adecuando a las exigencias que en el tiempo se manifiestan es muy importante. Objetivo: del presente artículo de reflexión es describir los aspectos sincrónicos y diacrónicos subyacentes al desarrollo del posgrado en Educación Especial de FESZ desde 1987 a la fecha. Se muestra la evolución del posgrado en el tiempo, las bases teóricas que lo sustentan, el análisis de la situación de maestros y de alumnos respecto del egreso, no egreso y nivel ocupacional. Discusión: Se reconoce el dinamismo desde su instrumentación, la relación que ha guardado con diversos factores en los que ha estado inmerso, tanto los administrativos y académicos propios de la institución como de otros externos: políticos, económicos y científicos. Se concluye con las aportaciones al campo de la educación especial logradas y las áreas de oportunidad tanto disciplinares como formativas ante los retos futuros.

Palabras clave: posgrado; sincronía; diacronía; educación especial; currículo.

\section{Synchrony and Diachrony of the Postgraduate Program in Special Education at the Faculty of Higher Studies Zaragoza (FESZ): Balance and Perspectives}

\footnotetext{
Abstract

Introduction: To design a postgraduate study plan is an relevant task for what is expected in the future about the training of its students. To implement it and to adapt it to the different demands that are manifested over time are major words. Objective: is to describe the synchronic and diachronic elements underlying the postgraduate program in Special Education at the FESZ since 1987 to date. It shows the evolution of the postgraduate course over time, the theoretical
} 
bases that support it, the situation teachers and students regarding graduation, no graduation and occupational level. Discussion: The dynamism from its implementation is recognized, the relationship that it has kept with various factors in which it has been immersed, both the administrative and academic of the institution as well as other external ones: political, economic, and scientific. It concludes with the contributions made to the field of special education and the areas of opportunity, both disciplinary and formative, in face of future changes.

Keywords: postgraduate; synchrony; diachrony; special education; curriculum

\section{Introducción}

Uno de los aspectos relevantes al implementar cualquier plan de estudios en el sector educativo del que se trate es reconocer el carácter dinámico que identifica al proceso de elaboración de forma sincrónica y diacrónica. Dinamismo que estará presente no solo en la instrumentación del programa mismo, sino en la relación que guardará con diversos factores en los que estará inmerso, tales como los administrativos y académicos propios de la institución que lo proponga como de otros externos: políticos, económicos y científicos. Este es el caso de la experiencia docente a la que se referirá este texto, centrado en la revisión del posgrado en educación especial de la Facultad de Estudios Superiores Zaragoza, posgrado pionero en este campo disciplinar en la Universidad Nacional Autónoma de México (UNAM).

\section{Aspectos Sincrónicos y Diacrónicos del Posgrado en Educación Especial}

El programa de Maestría en Psicología con orientación en Educación Especial se diseñó e implementó en 1984; en su fundamentación se consideraron las asignaturas de educación especial que se habían impartido desde 1978 en la licenciatura en Psicología de la FESZ, en las que se promovía que los estudiantes se capacitaran en las funciones profesionales de análisis, diseño, evaluación e intervención, que desempeñaban bajo supervisión al brindar servicios educativos especiales en las Clínicas Multidisciplinarias de Atención Inte- gral ubicadas en zonas metropolitanas de vulnerabilidad social. Aunado a lo anterior, se analizó la situación de la educación especial en México, tanto en los índices de demanda y atención brindados en aquel entonces por la Dirección General de Educación Especial de la Secretaría de Educación Pública (DGEE, SEP), como los programas afines al que se proponía (Acle-Tomasini, 1986).

La DGEE era la encargada de organizar, dirigir, desarrollar, administrar y vigilar el sistema federal de educación de niños con discapacidad con o sin necesidades educativas especiales, y también de la formación de maestros especialistas. A partir de su establecimiento en 1976, México se incorporó al grupo de países que de acuerdo con las recomendaciones de la Organización de las Naciones Unidas para la Educación (Unesco) reconoce a la educación especial como un área distintiva e indispensable en el conjunto de la educación básica general. Por lo tanto, en su labor integraba las políticas diseñadas y aprobadas en las reuniones de dicho organismo internacional. En aquel entonces, dichas políticas orientaban los servicios hacia la normalización y la integración (DGEE, 1981).

$\mathrm{Al}$ analizar los datos referentes a la población total del país, la demanda potencial de servicios de educación especial y la población realmente atendida en estos, se constató que si bien había un incremento en los niveles de atención del periodo de $1970-1971$ que era de .19\% al 1.8\% de 1982 1983, se estaba lejos de lograr atender al 10\% de una población que requería servicios de educación especial, tal como lo señalaba la Organización Mundial de la Salud en esa época (Acle, 1986). Los servicios que se brindaban estaban en: escuelas de educación especial, centros psicopedagógicos, centros de capacitación para el trabajo y las unidades de grupos integrados que atendían a los menores en pequeños grupos al interior de las escuelas. Asimismo, en 1980 en el área de posgrado existían solo dos programas de maestría que se brindaban en universidades privadas, ambos centrados en la categoría de problemas de aprendizaje. Por otro lado, la investigación que se hacía en educación especial en relación con otras áreas educativas era solo del 0.09\% (Galindo, 1987).

Con base en lo anterior, este posgrado buscó marcar una diferencia entre la formación que se 
daba en licenciatura, en la que el ejercicio profesional del psicólogo en el área de educación especial era el eje, mientras que el programa de maestría pretendía promover la formación en investigación para brindar respuestas a los múltiples problemas del campo de educación especial. En síntesis, se propuso que la investigación fuera el eje principal del plan de estudios para orientar al estudiante a la generación de nuevos marcos analíticos de las problemáticas y la producción de conocimientos teóricos y prácticos acordes con la realidad del país. Este programa fue aprobado por el Consejo Universitario de la UNAM en 1986 y se inició formalmente en 1987.

De forma sincrónica, a la instrumentación del plan de estudios, las políticas internacionales que se dictaban en encuentros como la Declaración Mundial de Educación para Todos realizada en Jomtien en 1990 y la Declaración de Salamanca en 1994, entre otros, marcaban la pauta para las modificaciones que habrían de ejecutarse para el establecimiento de políticas nacionales, normas y acciones de los países participantes, con el objetivo primordial de proporcionar una educación con calidad y equidad a todos aquellos estudiantes con requerimientos de educación especial. En México se modificó el artículo 41 de la Ley de Educación particular para educación especial, en él se estableció entre otros aspectos que se orientara a los maestros y al personal de escuelas de educación básica para integrar a los alumnos con necesidades educativas especiales en las aulas regulares.

Para ello, se reorganizaron los servicios públicos de educación especial, se establecieron: a) las Unidades de Servicio y Apoyo a la Escuela Regulares; b) los Centros de Atención Psicopedagógica de Educación Preescolar; c) los Centros de Atención Múltiple; d) los Centros Regionales de Integración Educativa; y, e) las Unidades de Orientación al Público. El postulado principal era integrar a los estudiantes de educación básica a un currículo común y evitar el tener otro paralelo (SEP, 2011). En el 2002 se estableció el Programa Nacional de Fortalecimiento de la Educación Especial y la Integración Educativa ([PNFEEIE], SEP, 2002), como la instancia que evaluaría los logros de la implantación de la política llamada en aquel entonces de integración educativa. Este siguió operando hasta 2018.
Lo antes señalado, se implementó en la formación de nuestros estudiantes de maestría, con miras al posible mercado laboral en que podrían insertarse al finalizar sus estudios. Así mismo, se buscaba profundizar en los avances científicos de la disciplina, tanto nacionales como internacionales a fin de sustentar sus proyectos para obtener el grado.

De forma análoga, en 1995, dentro de la política universitaria referente al posgrado, se propuso la creación de programas únicos de maestría y doctorado con el objetivo de optimizar los recursos con los que la UNAM contaba en el posgrado. Para lograr esta adecuación cabe señalar que en el diseño, revisión y evaluación de la nueva propuesta fue indispensable la participación colegiada de profesores de posgrado de las diferentes facultades en que había programas de maestría y doctorado en psicología. En el caso de la maestría, se propuso un programa profesionalizante de Residencia en el que se conjuntara tanto la experiencia generada por el desarrollo de las habilidades para realizar investigación aplicada como la adquisición de competencias de alta calidad en el conocimiento profesional de la disciplina, según fuese el área de aplicación de la psicología (Programa de Maestría y Doctorado en Psicología,1999). Es así como el programa de maestría vigente desde 1987 se adecuó y transformó en la Residencia en Educación Especial (Roque et al., 2001), programa aprobado por el Comité Académico del Programa de Maestría y Doctorado en 2001, año en que se inició su instrumentación. Posteriormente, este mismo Comité se propuso la tarea de revisar, adecuar y modificar el programa en 2015.

A partir de entonces y como se anota en la figura 1, en el desarrollo del programa se siguen considerando las políticas internacionales en las que México participa como miembro de la Unesco, como la Declaración de Incheon, en 2015, en el Foro Mundial de Educación 2015 y la Agenda para el Desarrollo Sostenible 2030 propuesta por la ONU en 2015, entre otros. Asimismo, se toman en cuenta los cambios de orden administrativo y político que operan en el subsistema oficial de educación especial y los avances científicos con respecto a tópicos fundamentales en el campo: a) el concepto teórico acerca de esta disciplina y las 
categorías que la conforman; b) los fundamentos teóricos prácticos de la evaluación de las necesidades educativas especiales y la discapacidad; c) los fundamentos teórico-prácticos para el diseño, instrumentación y evaluación de programas de intervención y prevención; d) elementos para el desarrollo de la investigación aplicada; $y, e)$ el conocimiento de las políticas nacionales e internacionales que rigen al campo (Acle-Tomasini, 1996).

Aunado a lo anterior, el Comité Académico del Programa de Maestría y Doctorado en Psicología sional de la psicología.

\section{Figura 1}

Evolución del Programa de Maestría en Educación Especial de la FESZ y Elementos Intervinientes a Través del Tiempo

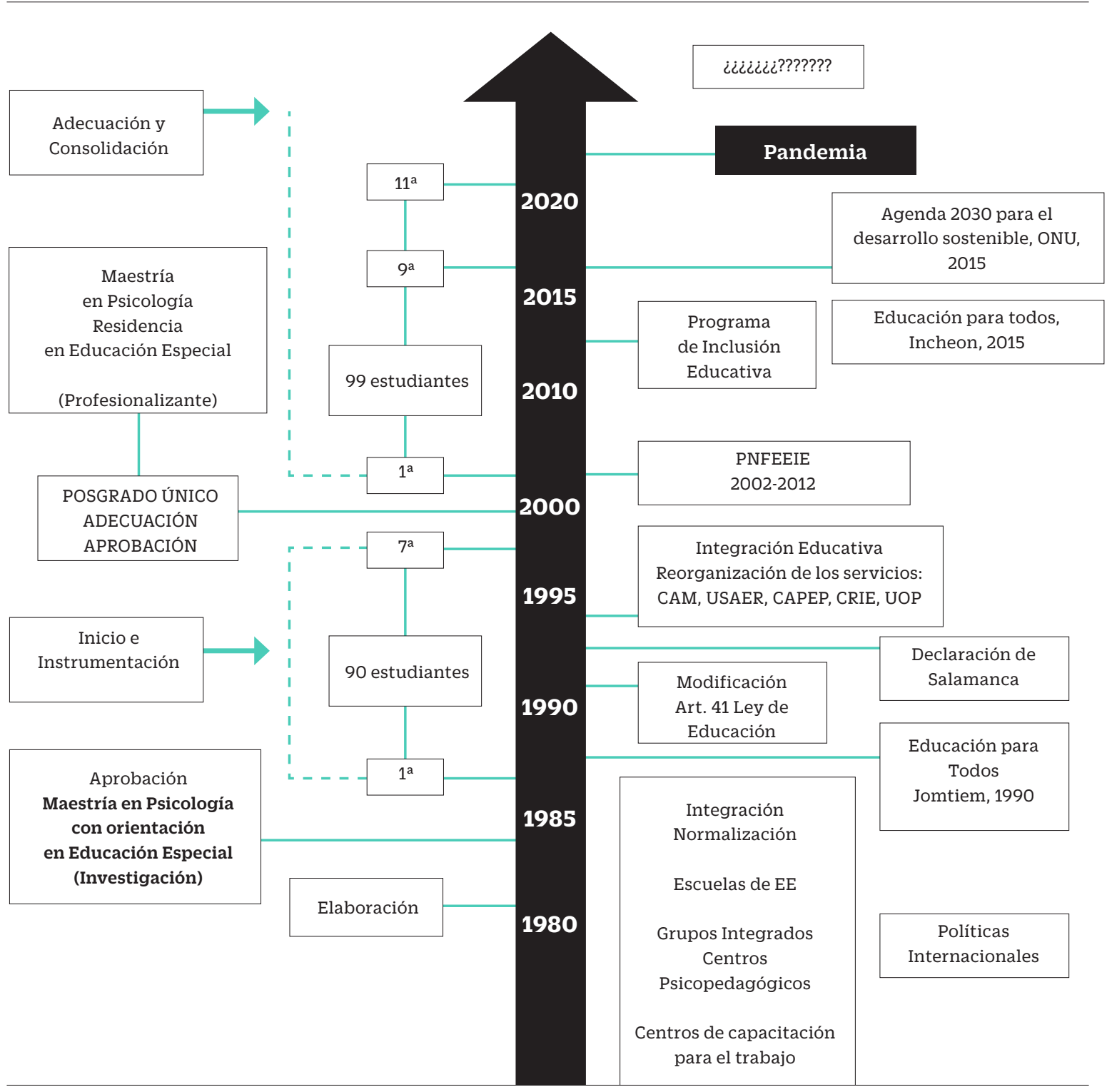

Nota. Elaboración propia 
Como se señaló al inicio, el dinamismo que se presenta en el devenir de un plan de estudios está influido de forma sincrónica y diacrónica por los factores tanto internos como externos al programa (Figura 1). Además de lo antes mencionado, encontramos lo imprevisto que ha sido para todos la pandemia y el confinamiento resultante, el cierre de actividades escolares nos planteó nuevos retos en la formación profesional y de investigación que se realizaban. Abrirse a las posibilidades de la educación en línea fue la opción, el desafío era cómo llevar a cabo la instrumentación de los programas de intervención que se habían iniciado en las escuelas básicas, a la vez de considerar las condiciones materiales y de recursos propios de la población foco, esto es, estudiantes provenientes de contextos de vulnerabilidad social.

A modo de síntesis, cabe señalar que este posgrado en educación especial ha ido evolucionando de forma tanto sincrónica como diacrónica, puede afirmarse que a través del tiempo se ha llevado a cabo en dos fases: a) Inicio e instrumentación (1987-2000) y, b) adecuación y consolidación (2001 a la fecha)

\section{Bases Teóricas del Currículo}

En primer lugar, es importante señalar que se parte de la base que la Educación Especial es una disciplina multidimensional, multiparadigmática y muiltidisciplinaria; los fundamentos del sistema de enseñanza modular brindan el soporte teórico y metodológico al programa de posgrado en ambas fases. Este sistema se caracteriza por cuatro aspectos primordiales:

1. Vinculación teoría-práctica: elemento fundamental para la construcción y aplicación del conocimiento, esta permite que los alumnos desarrollen las funciones y competencias profesionales que, en los ámbitos de educación especial, podrán incorporar en su ejercicio profesional futuro.

2. Interdisciplinariedad: el campo de la educación especial no es exclusivo de una sola disciplina, lo que tanto a nivel teórico como práctico plantea una serie de desafíos para buscar las soluciones que sean efectivas y eficaces a los problemas que enfrenta este campo.

3. Satisfacción de necesidades sociales. Diversos resultados de investigaciones (Acle-Tomasini, 2013; Sánchez et al., 2003) han mostrado que, en las estadísticas oficiales referentes a los servicios de educación especial, no están todos los que deberían estar y, quienes, por la falta de una clarificación del concepto de necesidades educativas especiales, de entre otros aspectos, los alumnos con discapacidad con o sin requerimientos de educación especial, están en riesgo de desertar de la escuela o de rezagarse de forma significativa. Por consiguiente, si bien se han reorganizado los servicios a la luz de las políticas nacionales e internacionales, persisten problemáticas que se requiere atender tanto en la población de niños y adolescentes con discapacidad con o sin necesidades educativas especiales como en los actores con los que interactúan en sus contextos familiar, escolar y social.

4. Integración Investigación-docencia-servicio: aspecto medular en la realización de las actividades del posgrado. La integración es el proceso a partir del cual se conforman en un todo la investigación, la docencia y el servicio compartiendo un objeto común y entretejiendo sus propios objetivos particulares. El servicio es el proceso en el cual los aspectos de diagnóstico, evaluación, prevención e intervención son fundamentales en la atención que se brinde a la población-foco en los escenarios reales. Mientras que la docencia es el proceso de transmisión de información en el que participan tanto el alumno como el maestro y en el que se lleva a cabo la construcción del conocimiento por ambos. Por último, la investigación será el proceso en el que la teoría, la técnica y el método se articularán en una unidad para construir el conocimiento, buscar las determinantes de un fenómeno y generar tecnologías (Acle-Tomasini \& González, 1988).

Estas características se han visto reflejadas de forma diferenciada en la organización curricular del posgrado en educación especial de ambas fases. 
Tabla 1

Organización Curricular de la Maestría en Psicología con Orientación en Educación Especial (1987-2000)

\begin{tabular}{lllll}
\hline \multicolumn{1}{c}{ Semestre } & & \multicolumn{3}{c}{ Módulos } \\
\hline $1^{\circ}$ & Desarrollo normal y & Análisis de & Rehabilitación & \\
$2^{\circ}$ & Anormal & Sistemas & & \\
$3^{\circ}$ & & Educativos & Metodología para Metodología \\
$4^{\circ}$ & & el Diseño y la & y Teoría de la \\
& & & Evaluación & Investigación \\
\hline
\end{tabular}

Nota. Elaboración propia

Tabla 2

Organización Curricular del Programa de Maestría en Psicología. Residencia en Educación Especial (2002 a la Fecha)

\begin{tabular}{llll}
\hline & Semestre & & Asignaturas \\
\hline $1^{\circ}$ & & Métodos de & Medición y \\
$2^{\circ}$ & Teorías y & Investigación & Evaluación \\
$3^{\circ}$ & Modelos de & Aplicada & \\
& intervención & & \\
$4^{\circ}$ & PRÁCTICAS & SUPERVISADAS & \\
\hline
\end{tabular}

Nota. Retomado del Programa de Maestría y Doctorado en Psicología, 1999.

En la fase del programa correspondiente al inicio e instrumentación, como se señaló previamente, la investigación constituía el eje principal del plan de estudios a fin de orientar al estudiante hacia la producción de conocimientos tanto teóricos como prácticos, a fin de coadyuvar en la solución de problemas detectados en el ámbito nacional. Además de poner en práctica las funciones profesionales del psicólogo: análisis, diseño, evaluación, intervención e investigación. Aspectos que se pueden observar en los módulos presentados en la tabla 1, el correspondiente a metodología y teoría de la investigación era el único de dicho plan que se cursaba desde el primero hasta el cuarto semestre y en cuyos productos presentados por los alumnos deberían integrarse los aprendizajes logrados en los otros módulos (Acle-Tomasini, 1986). La terminología empleada en la nominación de los módulos correspondía asimismo a la que se utilizaba en la década de los 80. Lo cual también se integraba en los programas de los módulos propuestos.

En la tabla 2, se muestra la estructura que corresponde a los programas de residencia que forman parte de la maestría en psicología de la
UNAM orientados a diversas áreas aplicadas de la psicología. Se propone la formación de profesionales altamente calificados que desempeñen las funciones profesionales de análisis, evaluación, intervención, prevención e investigación. De aquí que, el énfasis se da a las prácticas supervisadas del $1^{\circ}$ al $4^{\circ}$ semestre, en ellas se integra la enseñanza práctica con la teórica en escenarios reales. En la Residencia en Educación Especial éstas se llevan a cabo particularmente en escuelas públicas.

Se especifican las competencias profesionales: a) conocimiento de las teorías que sustentan la disciplina; b) elaboración y aplicación de técnicas de detección temprana y evaluación diferencial e integral; c) elaboración de programas de evaluación, intervención y prevención; d) generación de conocimientos y desarrollo de prácticas innovadoras a través de la investigación aplicada; $y, e)$ ejercicio crítico y ético de la profesión manteniendo siempre un compromiso social (Roque et al., 2001). El objetivo general se orienta a formar profesionales altamente capacitados quienes, a partir de la práctica supervisada en diversos escenarios, adquieran los conocimientos metodológicos, teó- 
Figura 2

Situación de los Alumnos Respecto a la Baja, Egreso, Graduación y no Graduación del Posgrado de Educación Especial en Ambas Fases de su Instrumentación

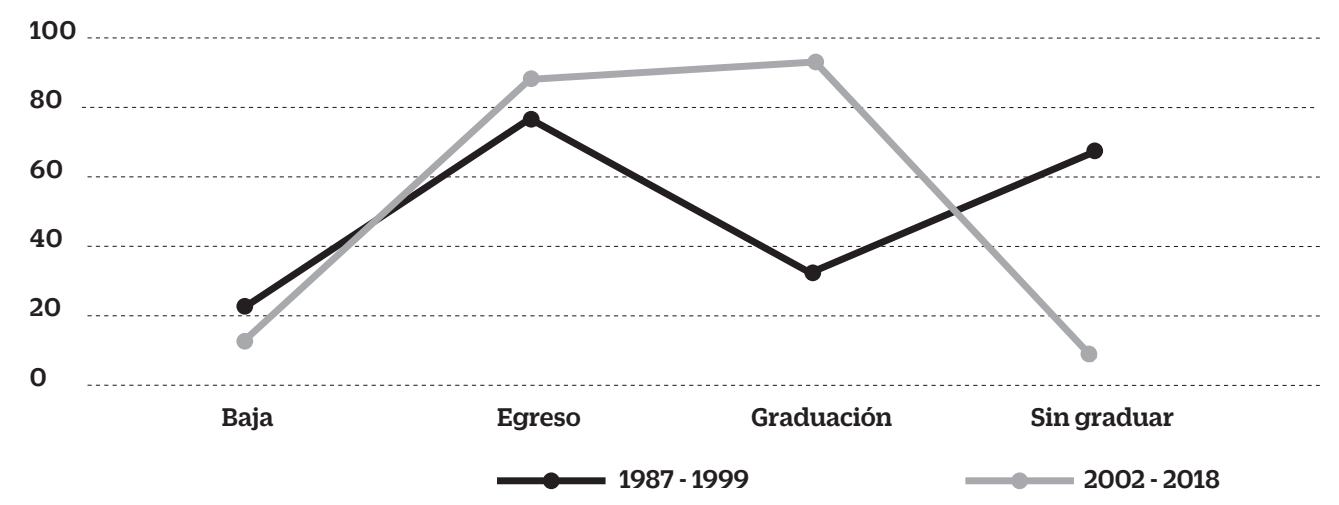

ricos y prácticos que les permitan realizar actividades de docencia, servicio e investigación e, incidir así, en la solución de los múltiples problemas presentes en el campo de la educación especial.

\section{Situación de Alumnos y Docentes}

Instrumentar un plan de estudios es todo un desafío, pues como se ha señalado existen tanto factores internos como externos al programa que de manera sincrónica y diacrónica están presentes. En lo que concierne a los internos, los actores que participan son fundamentales en el desarrollo de cualquier plan de estudios. El programa de la Maestría en Psicología con orientación en Educación Especial fue cursado por 90 alumnos que se ubicaron en siete generaciones de 1987 a 1999. En un inicio solo participaron tres docentes, por lo que se decidió abrir el programa de forma generacional, posteriormente al contar con el apoyo administrativo de directivos, fue posible incorporar como docentes del programa a cuatro alumnas egresadas del mismo. Mientras que, el programa de la Residencia en Educación Especial ha sido cursado de forma generacional por 114 alumnos de 2001 a la fecha, 99 de ellos se ubican de la primera a la novena generación y han completado sus estudios, de los 15 restantes, ocho de ellos están en trámites de titulación y siete están cursando actualmente el programa. La planta docente actual es de seis profesores, todos con estudios de doctorado.
Con respecto a los índices de egreso y graduación (Figura 2) se aprecian importantes diferencias en ambos programas, sobre todo en lo que concierne a los índices de graduación; de los 90 alumnos que cursaron el programa de 1987 a 1989, egresó el 77\% y de este porcentaje solo se graduó el 31\%, mientras que de los 99 alumnos que cursaron el programa de 2001 a 2016, del $87 \%$ que egresaron lograron su graduación el 92\%.

Esta diferencia se debe principalmente a diversos factores, entre ellos la falta de formación en investigación de los docentes al inicio del posgrado, quienes además eran pocos en relación con el incremento de los alumnos, aunado a factores socioeconómicos que llevaron a algunos de éstos a incorporarse inmediatamente al trabajo una vez terminada su formación u otros incluso a desertar. Además, en aquel entonces no era indispensable contar con el grado para insertarse en el mercado laboral. En este sentido, Herrera-Fragoso (2011) al llevar a cabo el seguimiento de egresados de estas generaciones encontró que el 57\% de ellos laboraba en el sector público y el 14\% en el privado, en ambos casos desempeñaban una o más de las actividades relacionadas con la docencia, la investigación y el servicio, algunos ocupaban puestos administrativos en la Secretaría de Educación Pública o en los servicios públicos del subsistema de educación especial (USAER o CAM). En general, los egresados de esta primera fase del posgrado señalaron que existía un reconocimiento a la formación obtenida independientemente de su situación laboral. 
Cabe también resaltar que de acuerdo con Herrera-Fragoso (2011) el 78\% de los participantes calificaron los contenidos teóricos del posgrado de buenos a excelentes, algunos indicaron que habían aprendido sobre investigación en contextos educativos. Ellos también señalaron la importancia de que el programa tuviera una mayor relación con instituciones públicas y privadas de educación especial, para realizar sus prácticas en escenarios reales. En cuanto a la investigación aplicada, solo el $47 \%$ indicó que pudo realizarla en contextos reales en los que desarrollaron las habilidades de evaluación e intervención, debido sobre todo a su participación de forma activa en la línea de investigación del tutor, en la que ellos realizaban su tesis para obtener el grado. Asimismo, el 75\% indicó que en proyectos futuros sería conveniente ampliar las temáticas de investigación en educación especial.

En lo que se refiere a la Residencia en Educación Especial, el porcentaje de egreso y de graduación se incrementó por diversos factores, uno de ellos fue que desde que se incorporaron a la planta docente egresados de este posgrado, por su propia formación y experiencia se favoreció la organización del programa, sobre todo lo relacionado con la estructuración de las actividades prácticas, ello facilitó el desempeño y desarrollo de las funciones profesionales consideradas en el objetivo general. A su vez, al pertenecer el programa al Padrón de Posgrados de Excelencia del Consejo Nacional de Ciencia y Tecnología, los estudiantes pudieron contar con una beca, con lo cual disminuyó la deserción por problemas económicos. Al irse consolidando las líneas de investigación de los tutores y contar con financiamiento de los proyectos, fundamentalmente del Programa de Apoyo a los Proyectos de Investigación e Innovación Tecnológica de la UNAM, se ha podido también apoyar económicamente a los estudiantes después de finalizar los estudios formales de la Residencia para obtener el grado en tiempo y forma de acuerdo con las normas establecidas en el programa. Elementos que han impactado favorablemente en una mayor graduación, de aquí que a esta fase le hemos denominado de adecuación y consolidación.

\section{Logros y Áreas de Oportunidad.}

Es importante señalar las contribuciones que en el campo de la educación especial se han tenido tanto en lo que concierne a la formación en el nivel de posgrado como en la disciplina misma. En cuanto a la vinculación teoría-práctica aunada a la integración de la investigación-docencia-servicio, cabe señalar que desde el inicio del programa de maestría se desarrollaron tres líneas de investigación que a la fecha se han consolidado: a) Modelo ecológico de riesgo resiliencia en ámbitos educativos y sociales (Acle-Tomasini et al., 2016); b) Promoción de conductas pro sociales y disminución del bullying en escuelas de educación básica (González-Granados et al. 2020); e, Identificación y diseño de programas de enriquecimiento para alumnos sobresalientes (Zacatelco-Ramírez, 2015).

Al integrarse los estudiantes a estas líneas de investigación a través de las actividades prácticas y de investigación aplicada que se realizan en escuelas públicas, se han caracterizado los factores individuales de los estudiantes de educación básica con requerimientos de educación especial y aptitud sobresaliente, habitantes de zonas de vulnerabilidad social (Hernández-Hernández, 2020; Martínez-Santos, 2020; Rivera-Ramírez, 2019; Martínez-Olivera, 1999); identificado los elementos contextuales familiares escolares y sociales que contribuyen a la erradicación o permanencia de las necesidades educativas especiales (López-Becerra, 2011; García-Olguín, 2021; Martínez-Maldonado, 1999).

Se ha coadyuvado asimismo en la solución de algunas problemáticas de menores detectados con diversos requerimientos de educación especial al interior de las escuelas regulares a través de los programas de evaluación, intervención e investigación aplicada que diseñan e instrumentan los estudiantes de la residencia. Entre ellos destacan: reducir el rezago escolar de alumnos de primer ciclo de primaria con necesidades educativas especiales (Barrera-Mares et al.; Rodea-Rodríguez, 2016; Romero-Muñoz, 2014); favorecer el desarrollo del potencial sobresaliente a partir de diversos programas de enriquecimiento (Gal- 
ván-Ruiz, 2021; González-Soria, 2017; Tepetla-Sánchez; 2015) y promover conductas prosociales en alumnos de secundaria a fin de disminuir conductas de acoso escolar (Soriano-Porto, 2012; Núñez-Hernández, 2018; Ortiz-Arroyo, 2016). Asimismo, se ha logrado involucrar a padres, maestros y directivos para favorecer la relación con sus hijos y alumnos respectivamente (Gutiérrez-Jiménez, 2004; Roque-Hernández, 2001, Villafranca-Fanyten, 2001), los autores antes citados son solo algunos ejemplos.

Desde que se inició este posgrado en educación especial en 1987 hasta la fecha se han actualizado los programas teóricos y prácticos al tomar en cuenta las políticas nacionales e internacionales que rigen el campo, el posible mercado laboral y los avances científicos de cada etapa del programa, todo lo cual ha favorecido nuestra participación en el logro de una inclusión educativa para que estos alumnos no sean excluidos al interior de las escuelas.

Con respecto al nivel de titulación de los alumnos se ha logrado un incremento sustancial, que a su vez ha facilitado a los egresados insertarse en el mercado laboral, cabe señalar que algunos de ellos se reincorporaron a sus labores en el subsistema oficial de Educación Especial, ubicándose incluso en puestos directivos. En fechas recientes, ocho egresados participaron en los Concursos de Oposición para el Ingreso a la Educación Básica del Sistema de Registro del Servicio Profesional Docente de la Secretaría de Educación Pública, a través de los que ganaron sus plazas y laboran por consiguiente en el subsistema oficial de Educación Especial. Otros egresados continuaron sus estudios en doctorado. Un aspecto benéfico para este posgrado ha sido la contratación como parte de la planta docente del posgrado a los egresados de este, en la primera fase del posgrado fueron cinco y de la segunda cuatro.

\section{Discusión}

Al considerar la naturaleza multidisciplinaria, multiparadigmática y multidimensional de la educación especial, el desarrollo y mejora de un plan de estudios de posgrado orientado en esta disciplina científica, no podrá dejar de tomar en cuenta de manera sincrónica y diacrónica los factores internos en relación a los recursos humanos y materiales para su desarrollo e instrumentación, así como los externos relacionados a las políticas nacionales e internacionales que rigen el campo y los avances científicos, que estarán inevitablemente inmersos en su evolución. Todo ello repercutirá indudablemente en los contenidos y las actividades curriculares que se propongan, el ejercicio profesional que se promueva y los proyectos de investigación que se desarrollen.

En este sentido, los retos que se nos plantean a nivel prospectivo, considerando la situación de pandemia que nos ha tocado vivir, implican tomar en cuenta lo establecido por la Unesco (2020) respecto de la importancia que tiene garantizar el acceso a los aprendizajes de los estudiantes en la situación del confinamiento, en particular aquellos que presentan discapacidad y necesidades educativas especiales. Un aspecto crucial es la necesidad de facilitar la vuelta de los estudiantes a las aulas cuando se pueda y evitar el abandono escolar, además de seguir brindando una educación de calidad. En este sentido, la participación de nuestros estudiantes ha sido decisiva, pues fue a través de medios virtuales que se realizó y brindó atención a aquellos menores que habían sido detectados con necesidades educativas especiales antes de la situación de confinamiento. Todo ello nos orienta para la generación de nuevos espacios a fin de indagar e investigar sobre lo que Gargiulo y Bouck (2021) denominan la Tecnología Asistida en Educación Especial.

El aprendizaje derivado de esta imprevista experiencia pone de manifiesto diversas áreas de oportunidad en la formación de nuestros estudiantes del posgrado tanto en sus actividades prácticas y de investigación aplicada. En lo que respecta a las primeras, se requirió diseñar e instrumentar programas híbridos de atención dirigidos tanto para alumnos con requerimientos de educación especial como para sus maestros y sus padres, quienes manifestaron que la situación de pandemia los había rebasado por la cantidad de trabajo, la reorganización de la enseñanza y de la vida diaria que se tuvo que hacer. Sobre todo, 
porque en el caso de los alumnos en situación de vulnerabilidad social, el acceso a los medios virtuales no fue fácil ni accesible (MEJOREDU, 2020). Otras áreas de oportunidad las marcan los objetivos de Educación de Calidad, Reducción de Desigualdades, Salud y Bienestar establecidos en la Agenda de Desarrollo Sostenible 2030 (ONU, 2015; Unesco, 2020), en los que se asienta, entre otros aspectos: facilitar la vuelta de los estudiantes a clases presenciales luego del confinamiento obligado, para evitar las tasas de deserción y rezago; buscar acciones equitativas y de acceso universal, así como atender no solo el bienestar físico de la población sino también la salud mental. Para lo que será relevante promover una atención interdisciplinaria ante las dificultades de salud física y emocional, educativas, económicas y sociales enfrentadas por directivos, maestros, alumnos con discapacidad con o sin necesidades educativas especiales y sus padres.

La educación especial como disciplina científica tiene mucho que ofrecer a la población que la requiere, de aquí que, en esta maestría centrada en la Residencia en Educación Especial de la Facultad de Estudios Superiores Zaragoza, será relevante seguir promoviendo una formación sólida en la que se considere la actualización permanente de los contenidos, la investigación aplicada aunada a un ejercicio profesional ético, crítico y comprometido. Ello implicará fomentar en nuestros estudiantes habilidades de pensamiento crítico y creativo que en el futuro les facilite enfrentar en su actividad profesional y de investigación diversas situaciones, sean estas imprevisibles o no. En este sentido, las bases del sistema de enseñanza modular, centradas en la vinculación teoría-práctica, la solución de problemas sociales, la interdisciplina y sobre todo, la integración de la investigación con la docencia y el servicio serán primordiales para generar nuevos marcos analíticos, producir conocimientos teóricos y prácticos acordes con la realidad del país para lograr lo que Kauffman et al. (2018) señalan, esto es no olvidar que la educación especial es fundamental en una sociedad democrática en la que se busque alcanzar el bienestar de todos sus ciudadanos.

\section{Referencias}

Acle-Tomasini, G. (1986). Plan de Estudios de la Maestría en Psicología con orientación en Educación Especial. ENEP Zaragoza, U.N.A.M. México. [manuscrito no publicado] Coordinación de Posgrado de la Facultad de Estudios Superiores Zaragoza. UNAM.

Acle-Tomasini, G. (1996). Vinculacion teoría-práctica: esencia de las prácticas de licenciatura y base de los estudios de posgrado en el área de Educación Especial. Revista del Consejo Nacional para la Enseñanza e Investigación en Psicología, Nueva Época, 1(2), 222-246.

Acle-Tomasini, G., \& González-Pineda. A. (1988). Integración de planes de estudio de Posgrado relacionados con el Desarrollo Humano. Propuesta de la ENEP Zaragoza. OMNIA, 4(11), 75-80.

Acle-Tomasini, G. (2013). Investigación en Educación Especial (2002-2011): Logros y desafíos. En R. De Agüero-Servín (Coord.), Aprendizaje y Desarrollo 2001-2011. (pp. 21-109). Consejo Mexicano de Investigación Educativa - ANUIES. http://www.comie.org.mx/v5/sitio/wp-content/uploads/2020/08/ Aprendizaje-y-desarrollo.pdf

Acle-Tomasini, G., Martínez-Basurto, L.M., \& Lozada-García, R. (2016). Bridging the gap between theory and practice: Ecological risk/resilience model contributions to special education. Journal of Creative Education, 7(7), 1093-1110. http://dx.doi.org/10.4236/ ce.2016.77114

Barrera-Mares, S.A., Acle-Tomasini, G., \& Martínez-Basurto, L.M. (2016). Modelo ecosistémico de riesgo/resiliencia: validez social de un programa para niños con problemas de lenguaje. En. J.L. Castejón-Costa (Coord.), Psicología y Educación: Presente y Futuro (2198-2206). Asociación Científica de Psicología y Educación.

Comisión Nacional para la Mejora Continua de la Educación (MEJOREDU, 2020). Experiencias de las comunidades educativas durante la contingencia sanitaria por covid-19. Educación Básica. MEJOREDU. https:// editorial.mejoredu.gob.mx/ResumenEjecutivo-experiencias.pdf

Comité Académico del Programa de Maestría y Doctorado en Psicología (2015). Proyecto de adecuación del programa de maestría y doctorado en Psicología. https://psicologia.posgrado.unam.mx/wp-content/uploads/2016/12/Plan-de-Estudios-PaCc81gina-Web.pdf

Dirección General de Educación Especial (1981). La educación especial en México. Cuaderno 8. Secretaría de Educación Pública.

Galindo, E. (1987). Necesidades de investigación en educación especial. Comunidad Zaragoza, 3(24). 
Galván-Ruiz, J.L. (2021). Club Comelibros: Programa de enriquecimiento en comprensión lectora para estudiantes con necesidades educativas especiales [Tesis de maestría]. Universidad Nacional Autónoma de México. http://132.248.9.195/ptd2021/enero/0806206/Index.html

García-Olguín, V. (2021). Promoción de la crianza materna como factor protector para infantes con retraso global del desarrollo desde un enfoque riesgo/resiliencia [Tesis de maestría]. Universidad Nacional Autónoma de México. http://132.248.9.195/ptd2021/ mayo/0811944/Index.html

Gargiulo, R.M., \& Bouck, E.C. (2021). Special Education in contemporary society. An introduction to exceptionality. SAGE.

González-Soria, A.D. (2017). Educación Emocional en alumnos con aptitud sobresaliente. [Tesis de maestría]. Universidad Nacional Autónoma de México. http://132.248.9.195/ptd2017/diciembre/0769137/ Index.html

González-Granados, A., Zacatelco-Ramirez F., \& Acle-Tomasini, G. (2020). Políticas antibullying en México. Contraste entre discursos políticos, acciones y resultados. En J. L. Crochick \& D. M. Avila de Lima (Coord.), Estudos entre violência escolar entre estudantes. Benjamin.

Gutiérrez-Jiménez, M.A. (2004). Programa dirigido a padres indígenas para estimular el desarrollo de sus hijos preescolares. [Tesis de maestría]. Universidad Nacional Autónoma de México. http://132.248.9.195/ ppt2004/0329990/Index.html

Hernández-Hernández, A.L. (2020). Perfiles cognitivos y académicos de niños con bajo rendimiento intelectual y su relación con los factores de riesgo [tesis de maestría]. Universidad Nacional Autónoma de México. http://132.248.9.195/ptd2019/diciembre/0799020/Index.html

Herrera-Fragoso, L. (2011). La maestría en Psicología (Educación Especial) de la FES Zaragoza: Seguimiento de Egresados [Tesis de maestría]. Universidad Nacional Autónoma de México. http://132.248.9.195/ ptd2012/enero/0676200/Index.html

Kauffman, J. M., Hallagan, D. P. Pullen, P. C., \& Badar, J. (2018). Special Education. What it is and why we need it. Routledge.

López-Becerra, M.L. (2011). Formas de interacción madre-hijo del nacimiento a los cuatro años. Un estudio con padres otomíes [Tesis de maestría]. Universidad Nacional Autónoma de México. http://132.248.9.195/ ptb2011/marzo/0667998/Index.html

Martínez-Maldonado, M.L. (1999). La percepción de maestros de una zona rural indígena acerca de los problemas de aprendizaje [Tesis de maestría]. Universidad Nacional Autónoma de México. http://132.248.9.195/pd1999/274940/Index.html

Martínez-Olivera, A.L. (1999). Problemas emocionales y de conducta en el aula: Una aproximación ecológica
[Tesis de maestría]. Universidad Nacional Autónoma de México. http://132.248.9.195/pd1999/270476/ Index.html

Martínez-Santos, B. (2020). Perfil cognitivo y académico de niños con problemas de lenguaje y su relación con factores de riesgo familiar y escolar [Tesis de maestría]. Universidad Nacional Autónoma de México. http://132.248.9.195/ptd2019/noviembre/0798463/ Index.html

Núñez-Hernández, D.E. (2018). Aula pacífica para reducir la violencia en niños con y sin aptitudes sobresalientes [Tesis de maestría]. Universidad Nacional Autónoma de México. http://132.248.9.195/ptd2019/ diciembre/0798871/Index.html

Organización de las Naciones Unidas (2015). Objetivos de Desarrollo Sostenible. https://www.un.org/sustainabledevelopment/es/2015/09/la-asamblea-general-adopta-la-agenda-2030-para-el-desarrollo-sostenible/

Organización de las Naciones Unidas para la Educación, la Ciencia y la Cultura (2020). Global Education Coalition https://en.unesco.org/covid19/educationresponse/globalcoalition

Ortiz-Arroyo, R.H. (2016). Apropiación de habilidades para la interacción en alumnos de primaria en riesgo [Tesis de maestría]. Universidad Nacional Autónoma de México.

Programa de Maestría y Doctorado (1999). Proyecto de adecuación: Programa de Maestría y Doctorado en Psicología. Universidad Nacional Autónoma de México.

Rivera-Ramírez, E.G. (2019). Diferencias de los perfiles cognitivos, socioemocionales y académicos de niños con problemas de conducta [Tesis de maestría]. Universidad Nacional Autónoma de México. http://132.248.9.195/ptd2019/noviembre/0798004/ Index.html

Rodea-Rodríguez, K. R. (2016). Modelo de atención para niños con problemas de conducta: Importancia de su validación social y educativa [Tesis de maestría]. Universidad Nacional Autónoma de México.

Romero-Muñoz, D.E. (2014). Expresión literaria: Estrategia educativa para promover la autorregulación en alumnos con problemas de conducta [Tesis de maestría]. Universidad Nacional Autónoma de México. http://132.248.9.195/ptd2014/anteriores/0715054/Index.html

Roque-Hernández, M.P. (2001). Estudio de la participación parental en una escuela monolingüe español de una comunidad rural e indígena otomí del Estado de México [Tesis de maestría]. Universidad Nacional Autónoma de México. http://132.248.9.195/ pd2001/296108/Index.html

Roque H. M.P., González, G. A., Zacatelco, R.F., Heres, P. J., \& Acle T.G. (2001). Plan de Estudios de la Residencia en Educación Especial. Adecuación de la Maestría en Psicología (Educación Especial) dentro de la Mae- 
stría en Psicología Profesional de la U.N.A.M. [manuscrito no publicado] Coordinación de Posgrado de la Facultad de Estudios Superiores Zaragoza. UNAM.

Sánchez. P., Acle, T.G., de Agüero, S.M., Jacobo, Z., \& Rivera, M. (2003) Estado del Conocimiento de la Investigación en Educación Especial en México (1990-2001). En P. Sánchez (Coord.), Aprendizaje y Desarrollo (pp.188-382). Consejo Mexicano de Investigación Educativa. http://www.comie.org.mx/doc/portal/ publicaciones/ec2002/ec2002_v04.pdf

Secretaría de Educación Pública (2002). Programa de Fortalecimiento de la Educación Especial y la Integración Educativa: SEP https://www.educacionespecial.sep.gob.mx/pdf/publicaciones/prognal.pdf

Secretaría de Educación Pública (2011). Modelo de atención de los servicios de educación especial. México.

Soriano-Porto, L.E. (2012). Propuesta para evaluar la apro- piación de la norma en alumnos de tercer ciclo de primaria [Tesis de maestría]. Universidad Nacional Autónoma de México. http://132.248.9.195/ptd2014/ anteriores/0713661/Index.html

Tepetla-Sánchez, F. (2015). El teatro: estrategia para favorecer la creatividad y el trabajo cooperativo en alum nos sobresalientes [Tesis de maestría]. Universidad Nacional Autónoma de México. http://132.248.9.195/ ptd2015/septiembre/0735436/Index.html

Villafranca-Fanyten, L. (2002). La vinculación de las variables: comunidad-escuela-familia-estudiante como predictora del riesgo escolar en alumnos otomíes de Zanja Vieja, Temoaya [Tesis de maestría]. Universidad Nacional Autónoma de México. http://132.248.9.195/pdtestdf/0302370/Index.html

Zacatelco-Ramírez, F. (Coord.) (2015). Atención educativa para alumnos de primaria con aptitudes sobresaliente. GEDISA-UNAM-FES Zaragoza.

RIDU / Revista Digital de Investigación en Docencia Universitaria / e-ISSN: 2223-2516

(c) Los autores. Este artículo es publicado por la Revista Digital de Investigación en Docencia Universitaria del Área de Institutional Research and Effectiveness de la Dirección de Aseguramiento de la Calidad, Universidad Peruana de Ciencias Aplicadas. . "Este es un artículo de acceso abierto, distribuido bajo los términos de la LicenciaCreativeCommons Atribución 4.0 Internacional (CC BY 4.0) (https://creativecommons.org/licenses/by/4.0/deed.es), que permite el uso, distribución y reproducción en cualquier medio, siempre que la obra original sea debidamente citada." 